EPiC Series in Computing
Volume 58, 2019, Pages 93-98
$\begin{gathered}\text { Proceedings of 34th International Confer- } \\ \text { ence on Computers and Their Applications }\end{gathered}$

\title{
An NC Algorithm for Sorting Real Numbers in $\boldsymbol{O}\left(\frac{n \log n}{\sqrt{\log \log n}}\right)$ Operations
}

\author{
Yijie Han, Sneha Mishra and Md Usman Gani Syed \\ School of Computing and Engineering \\ University of Missouri at Kansas City \\ Kansas City, MO 64110, USA \\ hanyij@umkc.edu, smccr@mail.umkc.edu, gmsc8c@mail.umkc.edu
}

\begin{abstract}
We apply the recent important result of serial sorting of real numbers in $O(n \sqrt{\log n})$ time to the design of a parallel algorithm for sorting real numbers in $\mathrm{O}\left(\log ^{1+\varepsilon} \mathrm{n}\right)$ time and $O\left(\frac{n \log n}{\sqrt{\log \log n}}\right)$ operations. This is the first NC algorithm known to take o(nlogn) operations for sorting real numbers.
\end{abstract}

Keywords: Parallel algorithms, sorting, sort real numbers, complexity.

\section{Introduction}

It is known widely that serial comparison sorting takes $\theta(\mathrm{nlogn})$ time [4]. Although integer sorting can outperform the $\Omega($ nlogn) lower bound for sorting integers [2,5,7,8,9,10], these algorithms generally do not apply to the problem of sorting real numbers. It has been known that integers can be sorted in $\mathrm{O}($ nloglogn) time and linear space $[7,8]$, the $\mathrm{O}(\mathrm{n} \operatorname{logn})$ time bound remains for sorting real numbers ever since. Only very recently Han showed that real numbers can be converted to integers for the sorting purpose in $O(n \sqrt{\log n})$ time [6], thus enabling the serial sorting of real numbers in $O(n \sqrt{\log n})$ time.

Parallel sorting algorithms for sorting real numbers run on the PRAM (Parallel Random Access Machine) model are known [1,3]. The AKS sorting network [1] can be transformed into an EREW (Exclusive Read Exclusive Write) PRAM algorithm with O(logn) time and O(nlogn) operations. Cole's parallel merge sort [3] sorts $n$ numbers in $\mathrm{O}(\log n)$ time using $\mathrm{n}$ processors on the EREW PRAM. On the CRCW (Concurrent Read Concurrent Write) PRAM Cole showed [3] that his parallel merge sort can run in $\mathrm{O}(\operatorname{logn} / \log \log (2 \mathrm{p} / \mathrm{n}))$ time using p processors. Also see [12]. 
There are also parallel algorithms for integer sorting $[2,5,7,8,9,10]$. In the case of integer sorting the operation bound can be improved to below $\mathrm{O}(\mathrm{n} \operatorname{logn})$. In particular, [9] presents a CRCW PRAM integer sorting algorithm with $\mathrm{O}(\log n)$ time and $\mathrm{O}(\mathrm{n} \log \log n)$ operations and [8] presents an EREW PRAM integer sorting algorithm with $\mathrm{O}(\log n)$ time and $O(n \sqrt{\log n})$ operations.

For sorting real numbers the previous best serial algorithm sorts in $\mathrm{O}(\mathrm{nlogn})$ time. It was also known that for comparison sorting $\Omega(\mathrm{n} \operatorname{logn})$ is the tight lower bound. Thus if we use comparison sorting to sort real numbers then in serial algorithms we cannot avoid the $\Omega(n \operatorname{logn})$ time bound and in parallel algorithms we cannot avoid the $\Omega(n \operatorname{logn})$ operation bound. In the past no other sorting methods are known to sort real number in less than $\mathrm{O}(\mathrm{n} \log n)$ time and comparison sorting remained the norm for sorting real numbers.

However the situation is recently changed completely as Han found a way to convert real numbers to integers for sorting purpose and he showed that real numbers can be sorted in $O(n \sqrt{\log n})$ time [6]. This result enables us to move further to improve the operation bound of parallel algorithms for sorting real numbers to below $\mathrm{O}(\mathrm{n} \operatorname{logn})$, as in the past all parallel algorithms for sorting real numbers has an operation bound at least $\mathrm{O}(\mathrm{n} \log )$.

In this paper we will apply the $O(n \sqrt{\log n})$ time serial real number sorting algorithm to the design of an NC algorithm with $\mathrm{O}\left(\log ^{1+\varepsilon} \mathrm{n}\right)$ time and $O\left(\frac{n \log n}{\sqrt{\log \log n}}\right)$ operations on the CREW (Concurrent Read Exclusive Write) PRAM. NC algorithms are parallel algorithms with polylog time and polynomial operations. Algorithm in [6] is an inherently serial algorithm without much parallelism within it. Here we use it in the design of an $\mathrm{NC}$ algorithm with $\mathrm{O}\left(\log ^{1+\varepsilon} \mathrm{n}\right)$ time and $O\left(\frac{n \log n}{\sqrt{\log \log n}}\right)$ operations.

The computation model used for designing our algorithm is the CREW PRAM. On this model in one step any processor can read/write any memory cell. Concurrent read of one memory cell by multiple processors in one step is allowed and concurrent write of one memory cell by multiple processors in one step is prohibited. Parallel algorithms can be measured with their time complexity and the number of processors used. They can also be measured with time complexity and operation complexity which is the time processor product. The operation complexity ( $T_{p} p$, with $T_{p}$ time using $p$ processors) of a parallel algorithm is often compared with the time $T_{1}$ of the best serial algorithm. In general $T_{p} p \geq T_{1}$. When $T_{p} p=T_{1}$ the parallel algorithm is said to be an operation optimal algorithm.

\section{The Algorithm}

Consider an algorithm for sorting $n$ real numbers. Suppose each of the $\mathrm{n} / \mathrm{m}$ lists with $\mathrm{m}$ real numbers in each list have already been sorted, we are to merge these $\mathrm{n} / \mathrm{m}$ lists into one sorted list. We will do kway merge in each pass to merge every k-lists into 1 sorted list and there $\operatorname{are~} \log (\mathrm{n} / \mathrm{m}) / \operatorname{logk}$ passes to have all $\mathrm{n} / \mathrm{m}$ lists merged into 1 sorted list.

For simplicity, let us break down $\mathrm{n}$ elements into lists with $\mathrm{m}$ elements in each list. We can do parallel sort on the individual list of $m$ elements recursively. Now we pick every $k$-lists and have them merged together.

The k-way merging of sorted lists $\mathrm{L}_{0}, \mathrm{~L}_{1}, \cdots, \mathrm{L}_{\mathrm{k}-1}$ is done as follows. For each sorted list of $\mathrm{m}$ real numbers we pick every $\mathrm{k}^{2}$-th real number, i.e. we pick the 0 th real number, the $\mathrm{k}^{2}$-th real number, the $2 \mathrm{k}^{2}$-th real number, the $3 \mathrm{k}^{2}$-th real number, and so on. Thus from each list $\mathrm{L}_{\mathrm{i}}$ we picked $\mathrm{m} / \mathrm{k}^{2}$ real numbers and these $\mathrm{m} / \mathrm{k}^{2}$ real numbers form a sorted list $\mathrm{L}_{\mathrm{i}}$. and from these $\mathrm{k}$ lists we picked $\mathrm{m} / \mathrm{k}$ real numbers they form sorted lists $\mathrm{L}_{0}{ }^{\prime}, \mathrm{L}_{1},{ }^{\prime}, . ., \mathrm{L}_{\mathrm{k}-1}$ '. We merge $\mathrm{L}_{0}, \mathrm{~L}_{1},{ }^{\prime}, ., \mathrm{L}_{\mathrm{k}-1}$ ' into one sorted list $\mathrm{L}^{\prime}$ using Valiant's merging algorithm [13] (its improved version is given by Kruskal in [11] with time complexity of $\mathrm{O}(\log \log \mathrm{m})$ and linear operations for merging two sorted lists of $\mathrm{m}$ elements each) in logk passes 
and $\mathrm{O}(\log \log \mathrm{m})$ time and $\mathrm{O}(\mathrm{m} / \mathrm{k})$ operations in each pass. Thus the total time for merging $\mathrm{L}_{0}, \mathrm{~L}_{1}, \ldots$ $\mathrm{L}_{\mathrm{k}-1}{ }^{\prime}$ is $\mathrm{O}(\operatorname{logk} \log \log \mathrm{m})$ and the total operation is $\mathrm{O}(\mathrm{m} \log \mathrm{k} / \mathrm{k})$.

Now for each real number $r$ in $L_{i}^{\prime}$ and for any $L_{j}{ }^{\prime}, r$ knows the largest real number $s$ in $L_{j}$ ' that is smaller than $r$ and smallest real number 1 in $L_{j}^{\prime}$ that is larger than $r$. $s$ and 1 are actually neighbors in $L_{j}$ '. There are $\mathrm{k}^{2}$ elements between $\mathrm{s}$ and $\mathrm{l}$ in $\mathrm{L}_{\mathrm{j}}$. $\mathrm{r}$ then uses binary search in $\mathrm{O}(\operatorname{logk})$ time to find the largest real number among these $\mathrm{k}^{2}$ real numbers that is smaller than $\mathrm{r}$ and the smallest real number that is larger than $r$. That is, $r$ finds the exact insertion point of $r$ in $\mathrm{L}_{j}$. Because there are $\mathrm{k}$-lists and there are $\mathrm{m} / \mathrm{k}$ real numbers in L' thus the time for this binary search is $\mathrm{O}(\operatorname{logk})$ and the operation is $\mathrm{O}(\mathrm{mlogk})$. The operation for all lists is $\mathrm{O}(\mathrm{n} \operatorname{logk} / \mathrm{k}$ ) because there are $\mathrm{n} / \mathrm{m}$ lists and every $\mathrm{k}$-lists are merged in the $\mathrm{k}$-way merge, we picked $\mathrm{n} / \mathrm{k}^{2}$ real numbers and every one of them has to use $\mathrm{k}$ processors to check $\mathrm{k}$ lists in the k-way merging. Because $r$ is arbitrary picked and thus we know that every real number in $L^{\prime}$ ' knows its insertion point in every $L_{j}$. Let the real numbers in sorted order in $L^{\prime}$ be $r_{0}, r_{1}, \cdots, r_{m / k-1}$. To merge $\mathrm{L}_{0}, \mathrm{~L}_{1}, \cdots, \mathrm{L}_{\mathrm{k}-1}$ we need now to merge or sort all real numbers between the insertion points of $r_{i}$ and $r_{i+1}$ in $L_{0}, L_{1}, \cdots, L_{k-1}$. There are no more than $k^{2}$ real numbers in $L_{j}$ between the insertion points of $r_{i}$ and $r_{i+1}$ and therefore the total number $R(i, i+1)$ of real numbers (call them a block) in $L_{0}, L_{1}, \cdots, L_{k-}$ 1 between the insertion points of $r_{i}$ and $r_{i+1}$ is no more than $k^{3}$ (i.e. $R(i, i+1) \leq k^{3}$ ). When $R(i, i+1)<k^{3}$ we will combine multiple blocks together to reach $\mathrm{k}^{3}$ real numbers. We use the $O(n \sqrt{\log n})$ serial sorting algorithm to sort them in $O\left(k^{3} \sqrt{\log k}\right)$ time. This represents $O\left(k^{3} \sqrt{\log k}\right)$ time and $O(n \sqrt{\log k})$ operations in our parallel algorithm.

Thus the time for each stage is $O\left(k^{3} \sqrt{\log k}\right)$ and the operation for each stage is $O(n \sqrt{\log k})$. When we start with $m$ as a constant then there are $\log / \operatorname{logk}$ stages and therefore the time of our algorithm is $O\left(\frac{k^{3} \log n}{\sqrt{\log k}}\right)$ and the operation is $O\left(\frac{n \log n}{\sqrt{\log k}}\right)$.

Pick k $=\log ^{\varepsilon}$ n, we get $\mathrm{O}\left(\log ^{1+\varepsilon} \mathrm{n}\right)$ time and $O\left(\frac{n \log n}{\sqrt{\log \log n}}\right)$ operations.

\section{Procedure}

Step 1: Lets say we have ' $m$ ' sorted elements in each list, and we have a total of 'n' elements to sort.

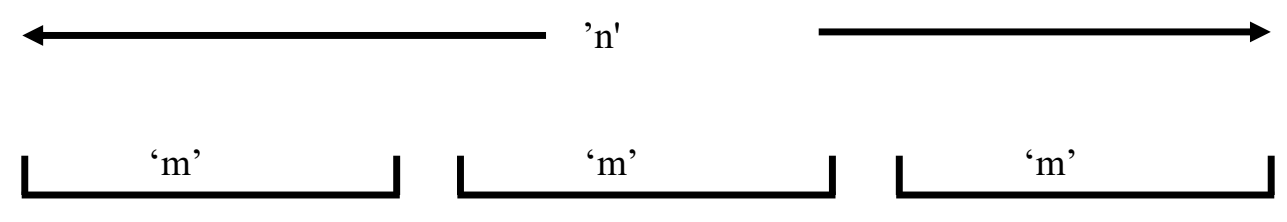

Figure 1

This implies that we have 'n/m' lists to sort. To sort these blocks, we will apply k-way merging. 
Step 2: Each stage of k-way merging is to merge every $\mathrm{k}$ sorted lists into 1 sorted list. This is repeatedly until all $\mathrm{n} / \mathrm{m}$ lists are merged into one list.

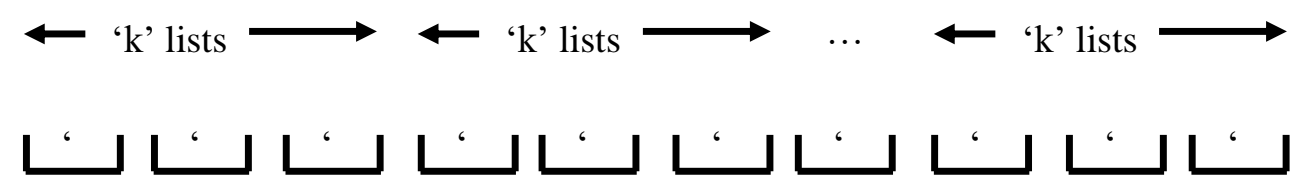

Figure 2

Step 3: To merge $k$ lists into 1 list, we need to pick the ' 0 -th', ' $k$-th', ' $2 k^{2}-$ th', ' $3 k^{2}$-th', .. real numbers in each list $\mathrm{L}_{\mathrm{i}}$ to form a new list $\mathrm{L}_{\mathrm{i}}$ ' of $\mathrm{m} / \mathrm{k}^{2}$ elements. This is shown as the figure below.

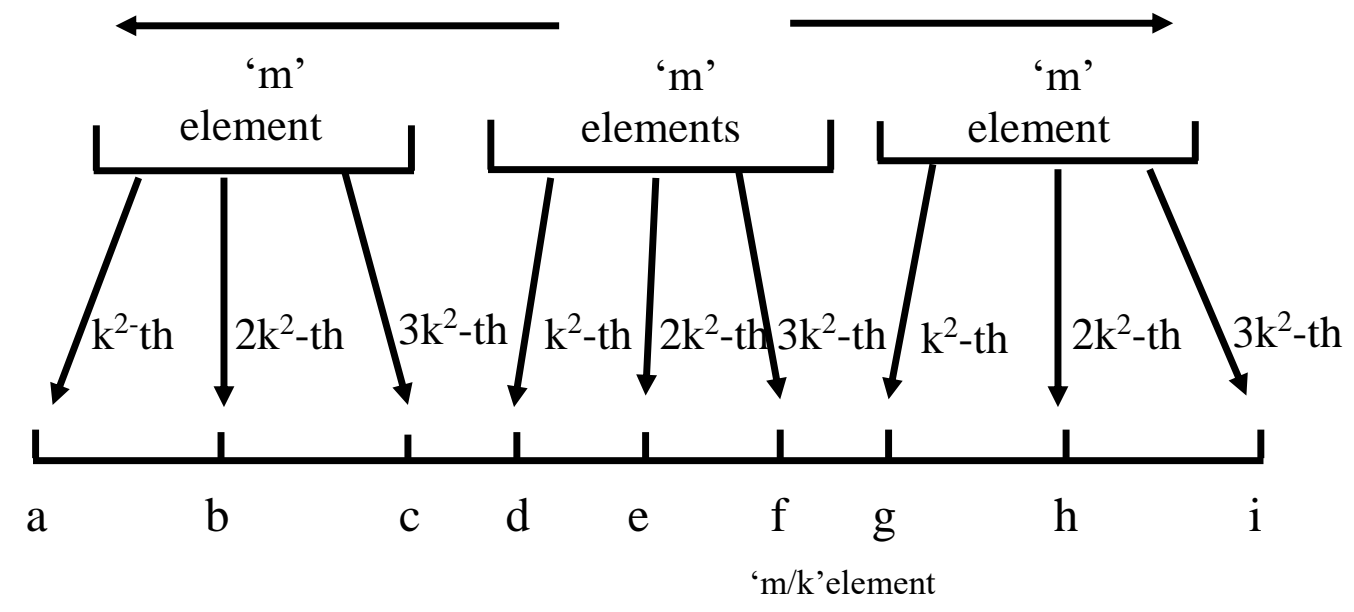

Figure 3

Step 4: We merge $\mathrm{L}_{0}, \mathrm{~L}_{1},{ }^{\prime}, . ., \mathrm{L}_{\mathrm{k}-1}$ ' into one sorted list $\mathrm{L}^{\prime}$. The elements of this new formed list $\mathrm{L}^{\prime}$ then use binary search to find their exact insertion point in $\mathrm{L}_{0}, \mathrm{~L}_{1}, \ldots, \mathrm{L}_{\mathrm{k}-1}$. These insertion points then partition $\mathrm{L}_{0}, \mathrm{~L}_{1}, \ldots, \mathrm{L}_{\mathrm{k}-1}$ into $\mathrm{m} / \mathrm{k}$ blocks with each block containing no more than $\mathrm{k}^{3}$ real numbers. 
Step 5: When every one of these $\mathrm{m} / \mathrm{k}$ blocks are sorted we effectively merged $\mathrm{L}_{0}, \mathrm{~L}_{1}, \ldots, \mathrm{L}_{\mathrm{k}-1}$ into one sorted list L.

Main Theorem: $\mathrm{n}$ real numbers can be sorted in $\mathrm{O}\left(\log ^{1+\varepsilon} \mathrm{n}\right)$ time and $O\left(\frac{n \log n}{\sqrt{\log \log n}}\right)$ operations on the CREW PRAM.

\section{References}

[1] A. Ajtai, J. Komlós, E. Szemerédi. 1983. An O(nlogn) sorting network. Proc. 1983 ACM Symp. On Theory of Computing (STOC'83), 1-9.

[2] P. C. P Bhatt, K. Diks, T. Hagerup, V. C. Prasad, T. Radzik, S. Saxena.1991. Improved deterministic parallel integer sorting. Information and Computation, 94, 1, 29-47.

[3] R. Cole. 1988. Parallel merge sort. SIAM J. Comput., Vol. 17, No. 4, 770-785(1988). Correction: Parallel merge sort. SIAM J. Comput., 22(6), 1349(1993).

[4] T. H. Corman, C. E. Leiserson, R. L. Rivest, C. Stein. 2009. Introductionto Algorithms. Third Edition, The MIT Press.

[5] T. Hagerup. 1987. Towards optimal parallel bucket sorting. Information and Computation, 73, 3951.

[6] Y. Han. 2017. Sort real numbers in $O(n \sqrt{\log n})$ time and linear space. In arXiv.org with paper id 1801.00776

[7] Y. Han. 2004. Deterministic sorting in O(nloglogn) time and linear space. Journal of Algorithms, 50, 96-105.

[8] Y. Han. 2015. A linear time algorithm for ordered partition. Proc. 2015 International Frontiers in Algorithmics Workshop (FAW'15), LNCS 9130, 89-103.

[9] Y. Han, X. Shen. 1995. Conservative algorithms for parallel and sequential integer sorting. Proc. 1995 International Computing and Combinatorics Conference, Lecture Notes in Computer Science 959, 324-333. 
[10] Y. Han, X. Shen. 2002. Parallel integer sorting is more efficient than parallel comparison sorting on exclusive write PRAMs. Proc. 1999 Tenth Annual ACM-SIAM Symposium on Discrete Algorithms (SODA'99), Baltimore, Maryland, 419-428(January 1999). Also in SIAM J. Comput.31, 6, 1852-1878.

[11] C. P. Kruskal. 1983. Searching, merging, and sorting in parallel computation. IEEE Trans. Comput., C-32, 942-946.

[12] S. Saxena, P. Chandra, P. Bhatt, V. C. Prasad, 1994. On parallel prefix computation. Parallel Processing Letters 4, 429-436.

[13] L. G. Valiant. 1975. Parallelism in comparison problems. SIAM J. Comput., 4, 348-355. 